\title{
Influência da pressão em propriedades de amostras nitretadas a plasma do aço inoxidável AISI 316L usado como biomaterial
}

\author{
Influence of pressure on properties of plasma nitrited samples of AISI $316 \mathrm{~L}$ \\ stainless steel used as biomaterial \\ Edilaine Honorio da Silva ${ }^{1}$, Marcelo Campos ${ }^{1}$, Sylvio Dionysio de Souza ${ }^{1}$, Maristela Olzon-Dionysio ${ }^{1}$
}

\begin{abstract}
Resumo
Este trabalho investiga a influência da pressão do processo de nitretação a plasma na modificação de algumas propriedades superficiais do aço inoxidável AISI 316L. As superfícies nitretadas foram analisadas por Difração de Raios X, Microscopia Eletrônica de Varredura e Microdureza. A nitretação a plasma foi realizada sob atmosfera de $80 \% \mathrm{H} 2$ e $20 \% \mathrm{~N} 2$, a $400^{\circ} \mathrm{C}$, durante $4 \mathrm{~h}$, com pressão entre 3 e 7 torr. Em todas as amostras observou-se a formação de anéis na borda da superfície nitretada, sendo que o aumento da pressão diminuiu a largura desses anéis. Além disso, no intervalo de pressão analisado, a largura d do anel formado é uma função decrescente de terceiro grau da pressão. A espessura da camada formada nas bordas, em todas as amostras, foi maior do que na região interna, e se torna mais uniforme e espessa quando a pressão aumenta. Alem disso, se a pressão cresce, a dureza média aumenta, bem como a difusão do Nitrogênio aumenta na camada nitretada.
\end{abstract}

Palavras-chave: Nitretação a plasma; Difração de raios-X; Microdureza vickers.

\begin{abstract}
The present work investigates the influence of gas nitriding pressure on the modification of surface properties of AISI $316 \mathrm{~L}$ stainless steel. Nitrided surfaces were characterized by X-ray diffraction, Scanning Electron Microscopy and Vickers microhardness. Plasma nitriding was carried out in a medium of $80 \% \mathrm{H} 2$ and $20 \% \mathrm{~N} 2$, at $400^{\circ} \mathrm{C}$, for $4 \mathrm{~h}$, pressures between 3 and 7 torr. In all samples some rings at the edge of the surface nitrided were observed. If the pressure increases, the width of these rings decreases. Moreover, in the pressure range examined, the width of the ring formed is a decreasing function of third degree of pressure. The thickness of the layer formed on the edges in all samples was higher than in the inner region, and it becomes more uniform and thicker when the pressure increases. Moreover, if the pressure increases, the average hardness and the diffusion of Nitrogen increase as well.
\end{abstract}

Keywords: Plasma nitriding; X-ray diffraction; Vickers microhardness. 


\section{Introdução}

Os aços inoxidáveis AISI 316L E ASTM F138 são amplamente usados, tanto em indústria quanto como biomaterial, por sua resistência à corrosão. Porém seu uso fica limitado devido à sua baixa dureza ${ }^{(1)}$.

Várias técnicas de modificação de superfícies vêm sendo desenvolvidas, capazes de melhorar as características tribológicas dos materiais. Entre as técnicas mais usadas está a nitretação a plasma, tanto por produzir amostras de aço inoxidável com maior dureza e também com resistência à corrosão ainda melhor, quanto por ser uma técnica de baixo custo e ecologicamente favorável, pois os resíduos não são tóxicos.

O processo de nitretação a plasma consiste na modificação da camada superficial do material, no nosso caso o aço inoxidável, formando nele diferentes fases contendo ferro e nitrogênio.

Entre as fases formadas em amostras de AISI 316L e ASTM F138, as principais são: a fase $\gamma \mathrm{N}$ (conhecida como fase fcc supersaturada de nitrogênio), a fase cúbica fcc $\gamma^{\prime}\left(\mathrm{Fe}_{4} \mathrm{~N}\right)$ e a fase hexagonal $\varepsilon\left(\mathrm{Fe}_{2}+\mathrm{xN}, 0 \leq \mathrm{x} \leq 1\right)^{(1)}$,e nitretos de cromo, que se formam para temperaturas acima de $420^{\circ} \mathrm{C}$.

Estudos recentes ${ }^{(2,3)}$ indicam que a formação das fases ferro-nitrogênio é mais sensível à variação da pressão do que dos outros parâmetros estudados, temperatura e tempo. Este trabalho analisa a influência da pressão em algumas das propriedades de amostras nitretadas de AISI 316L.

\section{Materiais e Métodos}

\section{Preparação das Asmostras}

Foram usinadas amostras de aço inoxidável AISI 316L, em formato de moeda, com 20,14 $\pm 0,02 \mathrm{~mm}$ de diâmetro e 2,00 $\pm 0,02 \mathrm{~mm}$ de altura. As amostras foram lixadas em lixas d'água, de grão entre 220 e 1200, depois foram polidas em suspensão de alumina de 1,0 e 0,3 $\mu \mathrm{m}$. A nitretação a plasma foi realizada sob atmosfera de $80 \% \mathrm{de}_{2}$ e $20 \%$ de $\mathrm{N}_{2}$, a $400^{\circ} \mathrm{C}$, durante 4 horas, com pressão entre 3 e 7 torr.

\section{Caracterização das Amostras}

Depois de nitretada, as amostras foram analisadas por Microscopia Eletrônica de Varredura (MEV), (equipamento
JEOL, modelo JSM - 5800 LV do DF-UFSCar); depois em um Microdurômetro, com carga de 35 gf para medida da dureza superficial, (modelo FISCHERSCOPE H100V do Laboratório de Fenômenos de Superfície, do Departamento de Engenharia Mecânica da Escola Politécnica da USP); e finalmente no Laboratório Nacional de Luz Síncrotron, (linha D12A-XRD1, $\lambda=1.900 \AA$ ), onde foram obtidos os difratogramas de raios-X.

\section{Resultados e Discussão}

\section{Análise Visual e MEV}

Em todas as amostras foi possível observar, a olho nu, a formação de um anel, de largura $d$, causado pelo efeito de borda. A Fig. 1 apresenta uma foto de uma amostra nitretada, onde é indicada a largura $d$ do anel formado.

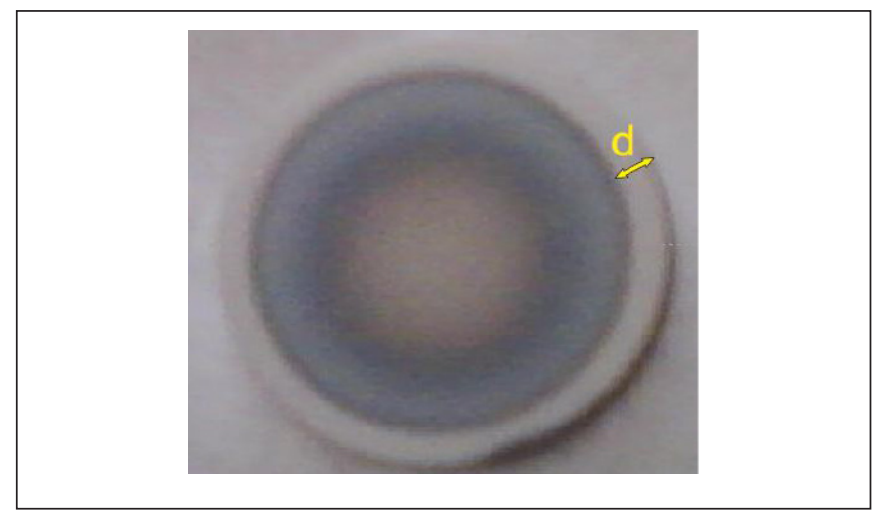

Figura 1. Região de formação do anel de largura $d$.

As imagens de MEV foram obtidas em três regiões: nas duas bordas $(0,00 \mathrm{~mm}$ e aproximadamente $20,00 \mathrm{~mm}) \mathrm{e}$ no centro das amostras (aproximadamente 10,00 mm). A Fig. 2 apresenta essas imagens para as amostras nitretadas a 3 e a 7 torr. Observa-se que para pressões maiores a espessura da camada nitretada é mais uniforme.

$\mathrm{Na}$ Tabela 1, tem-se a largura do anel formado $(d)$ e a espessura da camada formada para duas regiões da amostra: na borda da amostra (região do anel) e na região interna ao anel. A tabela traz também valores anteriores obtidos para AISI $316 L^{(1,2)}$ e ASTM F138 ${ }^{(3)}$, para comparação.

Analisando as segunda e quarta colunas da Tabela 1 observa-se que quanto maior a pressão usada, menor a largura do anel formado, o que também foi observado no aço ASTM F $138^{(3)}$. Vê-se também que no presente caso, a largura do anel é maior do que nos casos anteriores ${ }^{(1,2)}$, onde amostras de AISI 316L no mesmo formato de disco 

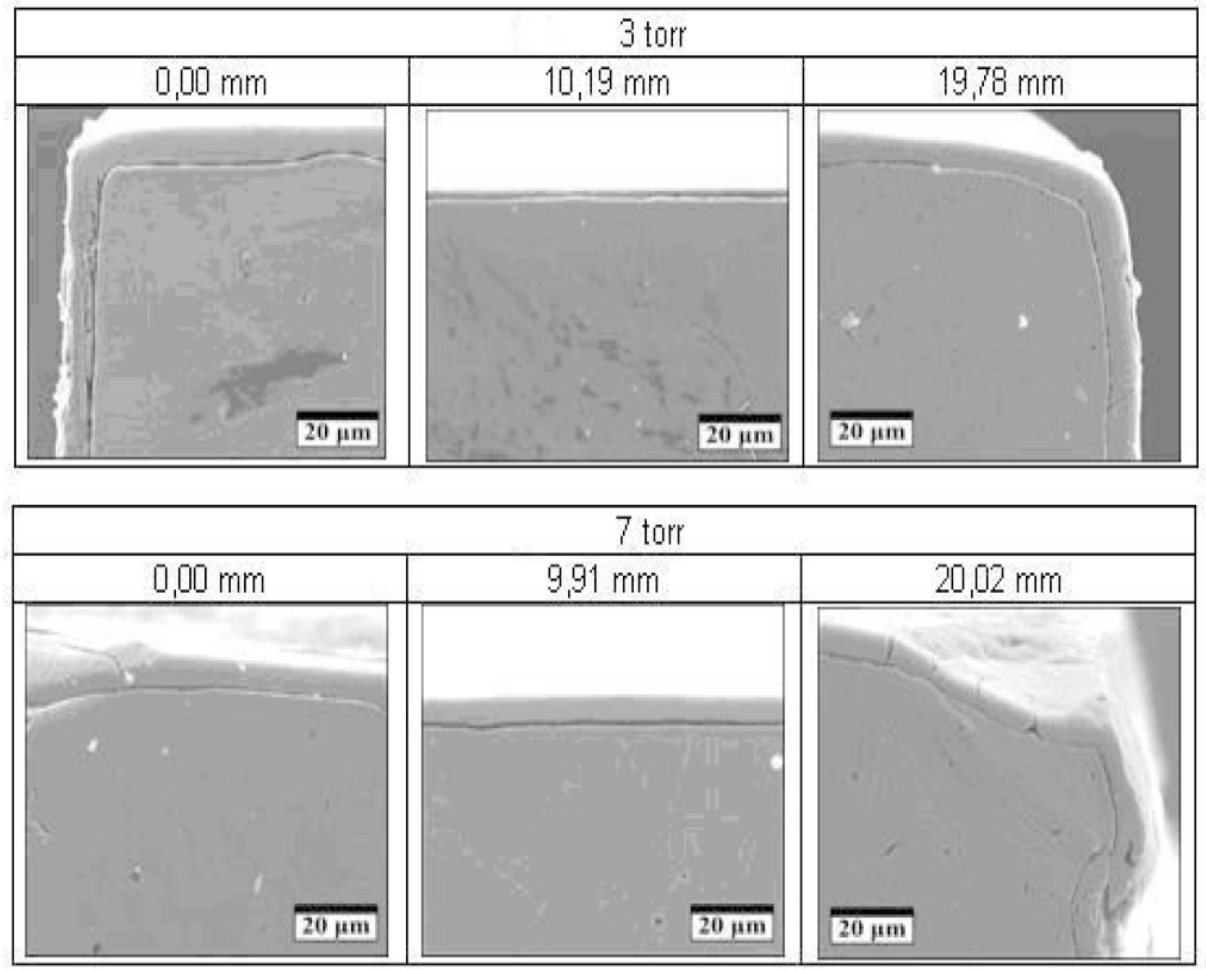

Figura 2. Imagens de MEV para amostras nitretadas a 3 e a 7 torr.

Tabela 1. Largura $d$ do anel e espessura da camada formada nas regiões interna ao anel e borda, onde este se forma.

\begin{tabular}{|c|c|c|c|c|c|c|}
\hline \multirow{3}{*}{$\begin{array}{c}\begin{array}{c}\text { Pressão } \\
\text { (torr) }\end{array} \\
3\end{array}$} & \multicolumn{3}{|c|}{ Largura do anel $(d)( \pm 0,02 \mathrm{~mm})$} & \multicolumn{3}{|c|}{ Espessura da camada formada $(\mu \mathrm{m})$} \\
\hline & \multicolumn{2}{|c|}{ AISI 316L } & \multirow{2}{*}{$\begin{array}{c}\text { ASTM F138 } \\
1,76\end{array}$} & \multirow{2}{*}{$\begin{array}{c}\text { AISI 316L } \\
\text { Na região interna ao anel } \\
1,7 \pm 0,2\end{array}$} & \multicolumn{2}{|c|}{$\begin{array}{c}\text { ASTM F138 } \\
\text { Na borda (região do anel) }\end{array}$} \\
\hline & 2,50 & & & & $6,8 \pm 0,9$ & 5,4 \\
\hline 4 & 1,70 & $1,31 \pm 0,06$ & 1,32 & $2,0 \pm 0,2$ & $6,7 \pm 0,6$ & 6,2 \\
\hline 5 & 1,22 & & 1,16 & $3,0 \pm 0,2$ & $9,1 \pm 0,7$ & 6,6 \\
\hline 6 & 0,92 & $0,65 \pm 0,03$ & - & $7,5 \pm 0,6$ & $8,6 \pm 0,9$ & - \\
\hline 7 & 0,64 & & 0,70 & $5,5 \pm 0,3$ & $8,9 \pm 0,9$ & 5,5 \\
\hline
\end{tabular}

foram usadas, mas com espessura maior, de praticamente o dobro, $3,90 \mathrm{~mm}$.

Ainda de acordo com a Tabela 1 , tem-se que a espessura da camada formada nas bordas, em todas as amostras, foi maior do que na região interna. Isso se deve ao efeito de borda, que distorce o campo elétrico na região das bordas ${ }^{(4)}$, fazendo com que a temperatura seja maior do que $400^{\circ} \mathrm{C}$, facilitando a difusão do nitrogênio e permitindo que a camada nitretada seja maior ${ }^{(1)}$. Vê-se ainda que a espessura da camada nitretada nas bordas das amostras não apresenta grandes variações
- a maior variação ocorre para 3 torr: $25 \%$ menor do que a máxima espessura, em 5 torr. No entanto, na região interna ao anel, a variação chega a ser grande, em relação à borda, chegando até a $75 \%$ para 3 torr. O menor valor, de $13 \%$ de diferença, acontece para 6 torr, que apresentou, assim, uma camada mais homogênea ao longo da superfície.

Em geral, a espessura da camada nitretada, na borda do aço ASTM F138, foi menor do que para 316L. A Fig. 3 ilustra o comportamento da largura do anel formado $(d)$ em função da pressão usada na nitretação. 


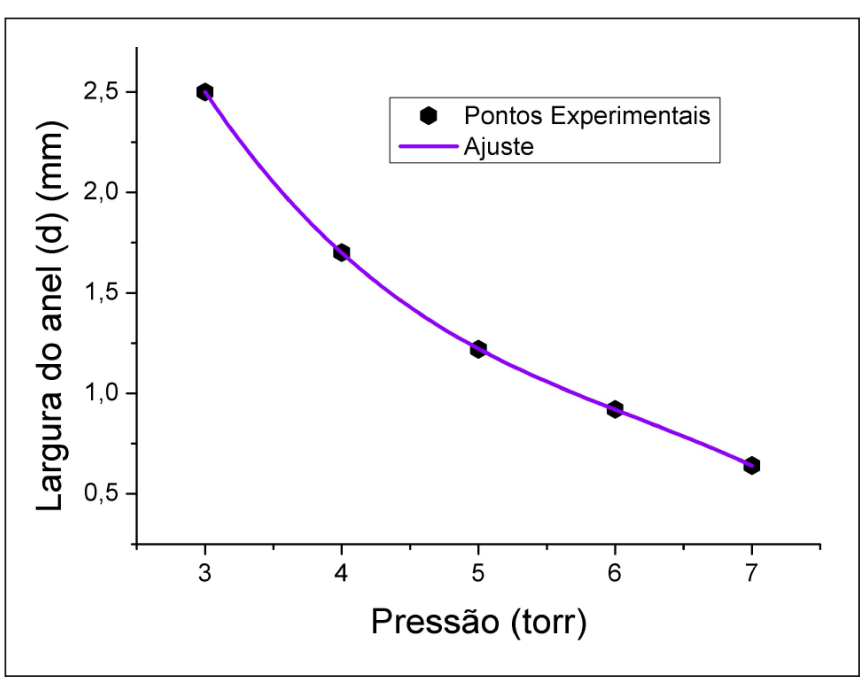

Figura 3. Largura do anel formado $(d)$ em função da pressão

A partir da Fig. 3 verifica-se que, no intervalo de pressão utilizado, a largura do anel formado no aço AISI $316 \mathrm{~L}$ se comporta como uma função decrescente de terceiro grau da pressão, cuja equação é dada por:

$$
d=8,350-3,111 \cdot p+0,462 \cdot p^{2}-0,025 \cdot p^{3}
$$

onde $d$ é a largura do anel formado e $p$ a pressão usada na nitretação.

\section{Medidas de Dureza}

A dureza Vickers foi medida na superfície das amostras, ao longo do raio. A Fig. 4 apresenta os valores medidos, como função da distância da borda, sendo que a linha vertical pontilhada indica o valor de d, largura do anel, para cada amostra.

As Fig. 4 (a), (b), (c), (d), (e) apresentam as curvas separadas das medidas de dureza para 3, 4, 5, 6 e 7 torr, respectivamente. Em cada uma delas foi calculada a média em diferentes regiões, na borda e na região central. Está assinalada a espessura do anel em cada caso, com uma linha vertical azul, pontilhada.

A Fig. 5 apresenta os valores médios calculados nas diferentes regiões das amostras, indicando também a largura do anel, com uma pequena reta vertical, na posição adequada. À direita são apresentados os valores médios na região central.

Para maior clareza, as amostras foram divididas em três regiões: anel, intermediária e central. Em qualquer dessas regiões a dureza das amostras é maior do que a da matriz, não nitretada. Nas regiões intermediária e central, a dureza aumenta quando a pressão cresce, embora a diferença seja maior na região central, aumentando quase de um fator 10 , para a pressão $\mathrm{P}=7$ torr. Nas pressões de 6 e 7 torr, Fig. 4(d) e 4(e), antes da região intermediária, a dureza apresenta uma queda brusca numa região bastante limitada, que não acontece para pressões menores. Nas regiões intermediária e central, a dureza aumenta quando a pressão cresce, embora a diferença seja maior na região central, aumentando quase de um fator 10 , para a pressão $\mathrm{P}=7$ torr. Nas pressões de 6 e 7 torr, Fig. 4(d) e 4(e), antes da região intermediária, a dureza apresenta uma queda brusca numa região bastante limitada, que não acontece para pressões menores.

A região do anel apresenta um valor maior de dureza, em relação à região intermediária, sendo que esta diferença diminui quando a pressão aumenta. Ainda na região do anel, a dureza aumenta para as amostras nitretadas, de 3 para 4 torr, e a partir de 5 torr, ela diminui, de modo que para 7 torr a dureza se aproxima muito do valor para 3 torr.

Comparemos agora os presentes resultados com valores obtidos anteriormente ${ }^{(2,5)}$.

A Tabela 2 apresenta os valores da dureza medida nas diferentes regiões para $\mathrm{P}=4$ (ou 3,7 ) torr e das dimensões das amostras correspondentes.

Examinando os valores desta tabela, podemos afirmar que a geometria das amostras exerce uma grande influência nos valores absolutos da dureza obtida. No entanto, nesse trabalho ${ }^{(2)}$ em que estudamos as características das amostras com anel, nitretadas ao mesmo tempo, demonstramos que, embora os diâmetros fossem diferentes, a razão entre os valores da dureza de regiões equivalentes apresentaram uma boa concordância. Assim, 652/206 $=3,2 \pm 0,7$ concorda com $1244 / 360=3,4 \pm 0,7$; bem como $431 / 206=2,1 \pm 0,4$ concorda com $775 / 360=2,2 \pm 0,4$.

Os resultados do presente trabalho apresentam $2213 / 481=4,6 \pm 1,3$ e $825 / 481=1,7 \pm 0,7$, respectivamente. Por outro lado, calculando essas razões para os resultados da última linha da Tabela 2, obtemos 1250/350=3,6 e $600 / 350=1,7$. Dessa forma, podemos afirmar que, levando-se em conta as barras de erro das razões calculadas, os resultados presentes estão em concordância com os obtidos anteriormente.

Borgioli e outros $^{(6)}$ mediram a dureza no perfil de amostras de AISI 316L (formato de prismas com 40x17x3 mm) tratadas a diferentes pressões, cujos valores estão dispostos na Tabela 3. A dureza da matriz concorda com a nossa, dentro de $8 \%$. No intervalo todo, sua dureza varia de $21 \%$. No entanto os autores não dão detalhes de suas medidas, 


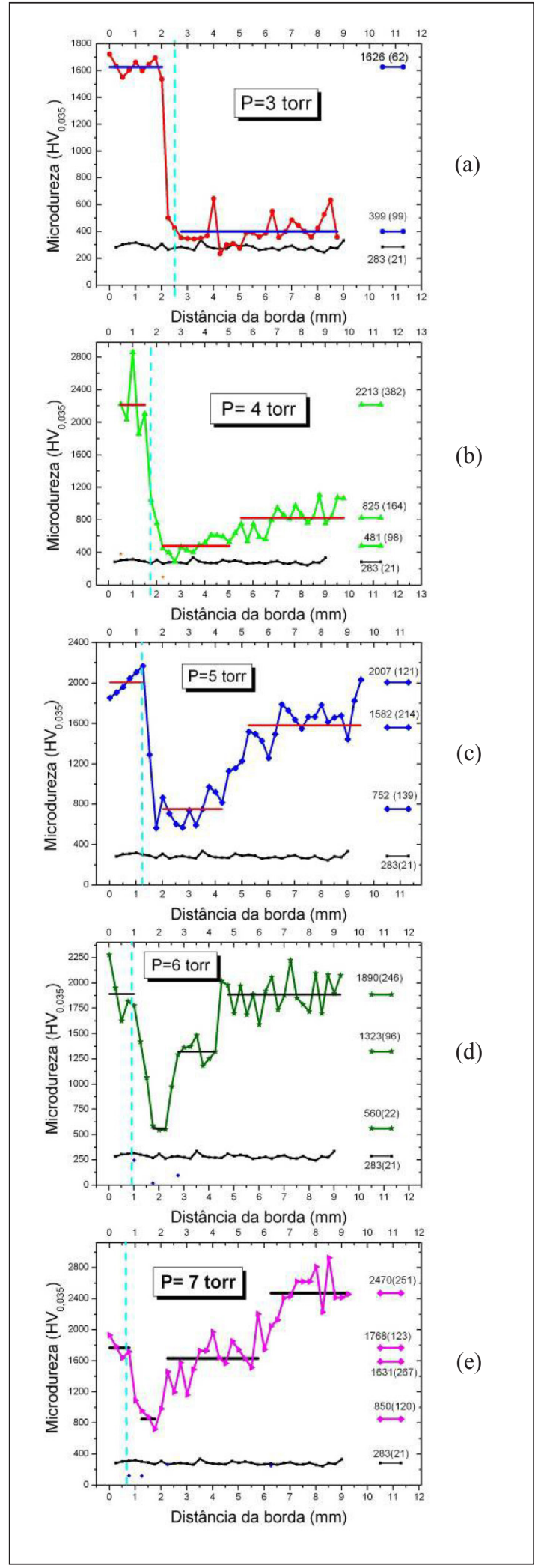

Figura 4. Medidas de dureza em função da distância até à borda, das amostras nitretadas a diferentes pressões (a)3, (b)4, (c)5, (d)6, (e)7 torr. Os valores médios calculados em diferentes regiões estão apresentados à direita, para cada um dos casos.

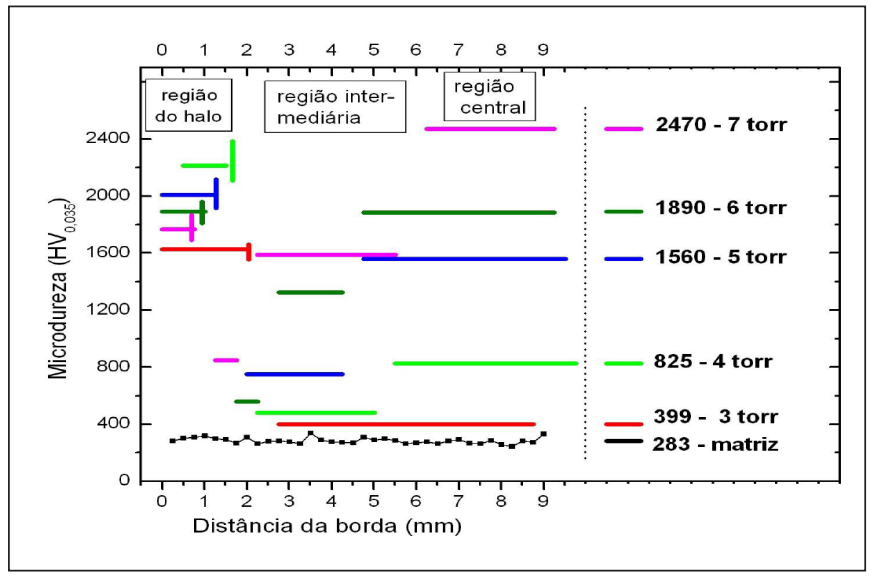

Figura 5. Valores médios da dureza em função da distância até à borda, das amostras nitretadas a diferentes pressões, nas diferentes regiões das amostras.

ou seja, não informam em que região mediram, se próximo da borda ou se internamente. Se observarmos seu resultado no intervalo que usamos (3,8 e 7,5 torr), a sua dureza variou de apenas $11 \%$. No presente caso, embora nossas medidas sejam na superfície, tomando-se $\mathrm{P}=4,0$ e 7,0 torr para comparação, vemos que a região que apresenta menor diferença neste caso é na borda, mas mesmo assim, sua variação é de $25 \%$. Desse modo, nossos resultados parecem não concordar com os desses autores. Pode ser que a geometria das amostras explique tal diferença.

\section{Difração de Raios-X}

A Fig. 6 mostra os difratogramas de raio-x das amostras com as posições das possíveis fases formadas.

Para 3 torr foi possível observar apenas os picos referentes à própria matriz, uma vez que a penetração máxima dos raios-x, que é de cerca de $10,0 \mu \mathrm{m}$, é muito maior do que a espessura da camada nitretada produzida, que é de 1,7 $\mu \mathrm{m}$. Em todas as outras amostras, observou-se a formação de um pico largo à esquerda do pico referente à matriz. Este pico é identificado como fase $\gamma_{N}$, que é a matriz supersaturada de nitrogênio e seu alargamento é devido ao gradiente de Nitrogênio, tensões residuais e possíveis defeitos criados na estrutura cristalina ${ }^{(7)}$. Além disso, quanto maior a quantidade de Nitrogênio, mais à esquerda esse pico está. Assim, vemos que o aumento da pressão resulta em maior difusão de Nitrogênio na amostra. Quanto às outras fases, $\varepsilon\left(\mathrm{Fe}_{2+\mathrm{x}} \mathrm{N}\right)$ e $\gamma^{\prime}\left(\mathrm{Fe}_{4} \mathrm{~N}\right)$, sua presença não pode ser observada por uma análise visual, pois seus picos ficam mascarados pelos picos largos da fase $\gamma_{\mathrm{N}}$. 
Tabela 2. Valores da dureza e das dimensões das amostras AISI 316L nitretadas nas mesmas condições de temperatura e tempo.

\begin{tabular}{c|c|c|c|c|c|} 
& $\begin{array}{c}\text { Diâmetro } \\
(\mathbf{m m})\end{array}$ & $\begin{array}{c}\text { Altura } \\
(\mathbf{m m})\end{array}$ & Borda & Região Intermediária & Região Central \\
\hline \multirow{2}{*}{$\operatorname{Refer}^{(2)}(\mathrm{P}=4$ torr $)$} & 19,70 & 3,90 & $652 \pm 70 \mathrm{HV}_{0,01}$ & $206 \pm 20 \mathrm{HV}_{0,01}$ & $431 \pm 40 \mathrm{HV}_{0,01}$ \\
\hline & 31,60 & 3,90 & $1244 \pm 90 \mathrm{HV}_{0,01}$ & $360 \pm 30 \mathrm{HV}_{0,01}$ & $775 \pm 80 \mathrm{HV}_{0,01}$ \\
\hline $\begin{array}{c}\text { Resultados deste trabalho } \\
(\mathrm{P}=4 \text { torr })\end{array}$ & 20,14 & 2,00 & $2213 \pm 382 \mathrm{HV}_{0,035}$ & $481 \pm 98 \mathrm{HV}_{0,035}$ & $825 \pm 164 \mathrm{HV}_{0,035}$ \\
\hline $\operatorname{Refer}^{(5)}(\mathrm{P}=3,7$ torr $)$ & 8,0 & 10,0 & $\approx 1200 \mathrm{HV}_{0,1}$ & $\approx 350 \mathrm{HV}_{0,1}$ & $\approx 600 \mathrm{HV}_{0,1}$ \\
\hline
\end{tabular}

Tabela 3. Resultados de Dureza para diferentes pressões de nitretação ${ }^{(7)}$

\begin{tabular}{|ccccccc|}
\hline Pressão(hPa) [torr] & --- & 1,5 & 2,5 & 5,0 & 10,0 & 20,0 \\
& Matriz & {$[1,1]$} & {$[1,9]$} & {$[3,8]$} & {$[7,5]$} & {$[15]$} \\
\hline Dureza $\left(\mathbf{H K}_{\mathbf{0 , 0 5}}\right.$ ) & $261 \pm 3$ & $1540 \pm 60$ & $1506 \pm 54$ & $1405 \pm 40$ & $1272 \pm 24$ & $1286 \pm 29$ \\
\hline
\end{tabular}

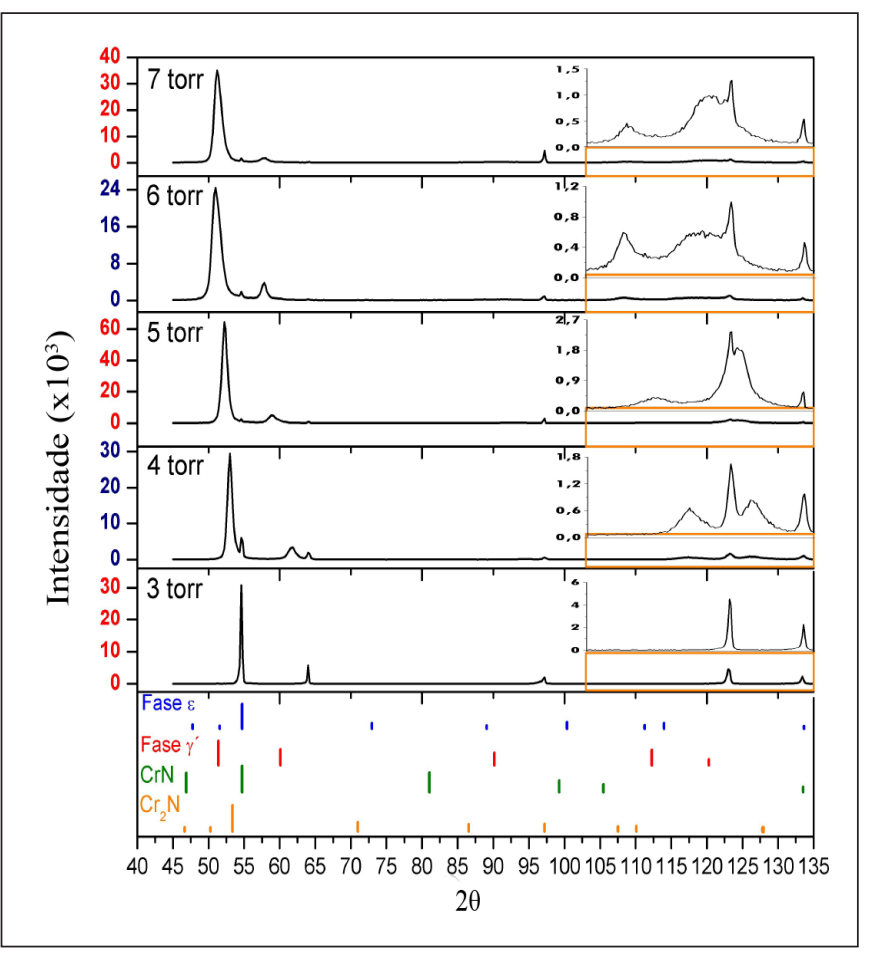

Figura 6. Difratogramas das amostras estudadas.

\section{Conclusão}

A partir da variação da pressão usada na nitretação a plasma foi possível chegar às seguintes conclusões:

- O aumento da pressão diminui o efeito de borda, uma vez que diminuiu a largura do anel formado. Além disso, verificou-se que, no intervalo de pressão analisado, a largura d do anel formado é uma função decrescente de terceiro grau da pressão para o AISI 316L;

- O valor médio da dureza aumenta com o aumento da pressão, independente da espessura da camada formada;

- O aumento da pressão tende a uniformizar a espessura da camada nitretada;

- O aumento da pressão resulta em maior difusão de Nitrogênio na amostra.

\section{Agradecimentos}

Os autores agradecem o apoio de Jefferson D. Alves e Thiago Pavan, durante a preparação das amostras; de Natália A. Zanardi, Leandro J. de Paula e Dr. L. M. Gallego no apoio durante as medidas realizadas de MEV, Dureza e DRX, respectivamente. Agradecemos ainda ao apoio do Laboratório Nacional de Luz Síncrotron e também às agências: CAPES, PIBIC/CNPq - UFSCar e FAPESP.

\section{Referências}

1. Campos, M., Investigação por Espectroscopia Mössbauer das fases formadas e sua influência na resistência à corrosão de aço inoxidável austenítico AISI 316L nitretado. Dissertação de Mestrado (2009). Universidade Federal de São Carlos, São Carlos (SP).

2. Olzon-Dionysio, M.; Campos, M.; Kapp, M.; De Souza, S; de Souza, S.D., Surface and Coatings Technology 204 (2010) 3623. 
3. Souza, S. D.; Kapp, M.; Olzon-Dionysio, M.; Campos, M., Surface and Coatings Technology 204 (2010) 2976.

4. Nishimoto A.; Nagatsuka K.; Narita R.; Nii R.; Akamatsu K, Surface and Coatings Technology (2010) no prelo: 10.1016/j.surfcoat.2010.08.034.

5. Alves Jr., C.; de Araújo, F. O.; Ribeiro, K. J. B.; da Costa,
J. A. P.; Sousa, R. R. M.; de Sousa, R. S., Surface and Coatings Technology 201 (2006) 2450.

6. Borgioli, F.; Fossati, A.; Galvanetto, E.; Bacci, T.; Pradelli, G., Surface and Coatings Technology 200 (2006) 5505.

7. Wang, L.; JI, S.; Sun, J., Surface and Coatings Technology 200 (2006) 5067. 